\title{
Carcinoma micropapilar de vejiga: aportación de un caso y revisión de la bibliografía
}

\author{
L. Ripa Saldías, R. Guarch Troyas*, A. Hualde Alfaro, A. De Pablo Cárdenas, M. Pinós Paul, \\ A. Santiago González de Garibay
}

Servicio de Urología y Servicio de Anatomía Patológica*. Hospital Virgen de Camino Pamplona.

Actas Urol Esp 2005; 29 (4): 408-413

\section{RESUMEN}

\section{CARCINOMA MICROPAPILAR DE VEJIGA: APORTACIÓN DE UN CASO Y REVISIÓN DE LA BIBLIOGRAFÍA}

El carcinoma micropapilar (CMP) es una variante anatomopatológica infrecuente de carcinoma vesical de comportamiento agresivo. Se presenta habitualmente como carcinoma de alto grado, en estadios avanzados y asociado a otras formas histológicas en proporciones variables. No manifiesta signos clínicos diferenciales con el carcinoma vesical típico. Los estudios de marcadores moleculares son todavía contradictorios. El tratamiento debería ser precoz y agresivo, fundamentalmente quirúrgico, dado que la Radioterapia y la Guimioterapia han demostrado escasa eficacia hasta el momento.

Presentamos el caso de un varón de 72 años con síntomas miccionales de larga evolución y hematuria macroscópica de reciente aparición que se diagnosticó de CMP en estadio avanzado. Al año de la cistectomía radical asociada a quimioterapia con carboplatino y gemcitabina se evidenció progresión rápida de la enfermedad y falleció a los 14 meses.

Palabras clave: Vejiga. Carcinoma transicional. Carcinoma micropapilar.

\section{ABSTRACT \\ MICROPAPILLARY CARCINOMA OF THE BLADDER: CASE REPORT AND REVIEW OF THE LITERATURE}

Micropapillary carcinoma is an uncommon pathologic variant of bladder carcinoma with aggressive behavior. Its usual presentation is like a high grade and high stage carcinoma and associated with other histologic types in different proportion. It doesn't differ clinically from normal transitional cell carcinoma of the bladder. Studies of molecular markers are still contradictories. Treatment should be early and aggresive, based on surgical therapy as radiotherapy and chemotherapy have shown limited results.

We report a 72 years old man suffering from low urinary tract symptoms for years and recently presented gross hematuria. He was diagnosed as high stage micropapillary carcinoma. One year after radical cystectomy and subsequent chemotherapy based on carboplatin and gemcitabine , progression of the disease was shown on CT and the patient died 14 months after the diagnosis.

Keywords: Bladder. Transitional carcinoma. Micropapillary carcinoma.

$\mathrm{E}^{1}$ carcinoma vesical presenta frecuentes variantes anatomopatológicas con implicación pronóstica. Una de las últimamente definidas es el carcinoma micropapilar (CMP), descrito por primera vez en 1994 por Amin ${ }^{1}$. Desde entonces se han comunicado pequeñas series y casos aislados de este tipo de tumor que demuestran el comportamiento agresivo y la escasa respuesta a tratamientos complementarios.

Lesiones histológicas micropapilares se han descrito en otros órganos e implican igualmente mal pronóstico, siendo en ocasiones dificultosa la 
determinación del origen primario cuando existen varios focos. Los estudios inmunohistoquímicos pueden orientar hacia el tumor primario.

El hallazgo de lesiones micropapilares en muestras de pared vesical o la presencia de papilas y células con anomalías nucleares muy llamativas en citologías urinarias nos deben alertar y completar los estudios de filiación, siendo imprescindibles las biopsias en profundidad de pared vesical, incluso en casos de lesiones planas, dada la capacidad infiltrativa de este tumor y por tanto su comportamiento agresivo.

\section{CASO CLÍNICO}

Paciente de 72 años con antecedentes de patología cardiovascular grave: cardiopatía isquémica con infarto de miocardio que precisó triple by-pass aorto-coronario, arteriosclerosis de troncos supraaórticos y de extremidades inferiores; EPOC, fumador hasta hace 2 años, hernia discal y estenosis de canal. Consultó por síntomas de tramo urinario inferior de un año de evolución, destacando micción nocturna de 4-5 veces/noche y polaquiuria diurna con micción en dos tiempos. Días antes de la consulta presentó hematuria total con coágulos. A la exploración física se apreciaba paciente con deterioro del estado general, abdomen globuloso y cicatriz de toracotomía. Al tacto rectal se palpaba próstata compatible con hiperplasia benigna grado II.

La analitica de sangre mostraba hemograma normal, función renal normal, leve hiperuricemia de 7,4 y PSA 0,9 $\mathrm{ng} / \mathrm{ml}$.

La ecografía inicial mostraba riñones normales y lesión exofítica en base vesical-cara derecha. La citología fue positiva para células malignas y la cistoscopia confirmó la lesión neoplásica vesical. El análisis de fragmentos tras RTU mostró carcinoma mixto, papilar de células transicionales grado II-III infiltrante de músculo liso con áreas de adenocarcinoma mucoide infiltrante asociado a lesiones de carcinoma in situ y cistitis glandular quística (estadio pT2-pT3 G-III).

El TAC posterior mostraba únicamente el engrosamiento de pared vesical y ateromatosis calcificada aorto-ilio-femoral siendo el resto de vísceras normales, sin detectarse adenopatías significativas.
Se realizó cistoprostatectomía radical y neovejiga ileal tipo Camey II. El postoperatorio cursó sin complicaciones y fue dado de alta al $10^{\circ}$ día.

El análisis histológico definitivo de la pieza revelaba una tumoración epitelial ulcerada infiltrante de todo el espesor de pared hasta tejido adiposo perivesical, constituida por nidos pequeños de células uroteliales dispuestas en lagunas artefactuales (Fig. 1), con imágenes concluyentes de invasión vascular (Fig. 2), con formaciones papilares sin tallo, que recuerdan al carcinoma seroso ovárico (Fig. 3). No se observaban cuerpos de psammoma. Había además lesiones asociadas de displasia epitelial en uretra prostática y un carcinoma de próstata bien diferenciado en lóbulo derecho Gleason $4(2+2)$. En pieza de linfadenectomía izquierda se aislaron 11 ganglios afectados por carcinoma micropapilar mientras que los ganglios linfáticos contralaterales no estaban afectados.

Inmunofenotípicamente el componente micropapilar expresó citoqueratina 7, CA125, citoqueratina 20 , BX2 y P53, siendo negativo para CEA.

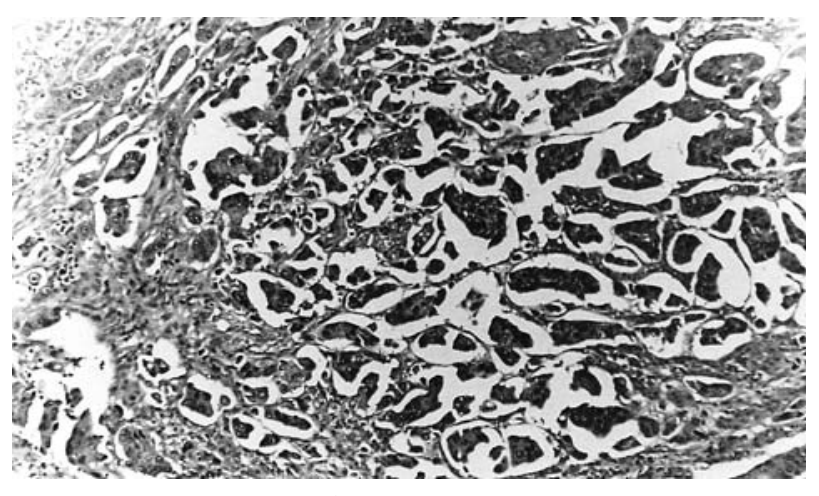

FIGURA 1. Nidos de células epiteliales que infiltran la pared vesical, dentro de hendiduras artefactuales.

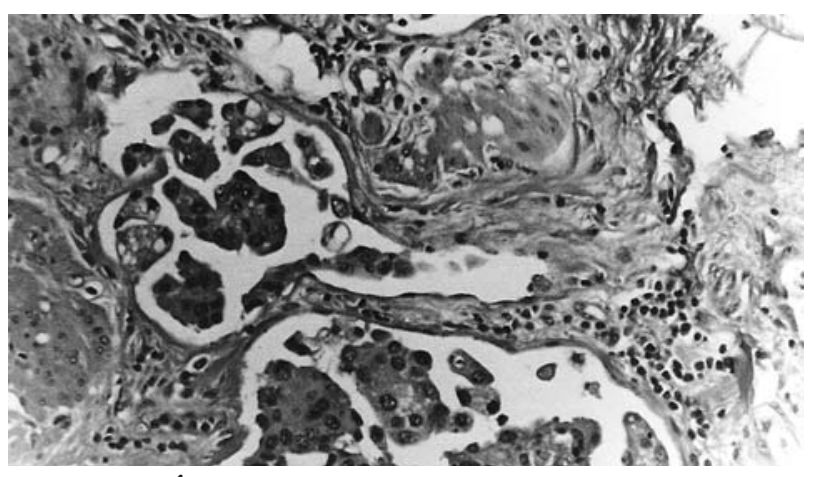

FIGURA 2. Áreas de infiltración de la pared, caracteristicas de este tumor, con áreas de invasión vascular. 


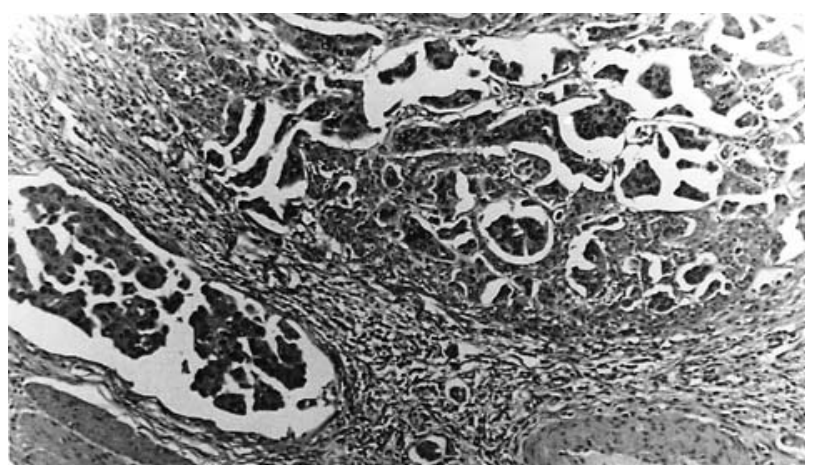

FIGURA 3. Invasión vascular por el tumor con crecimiento papilar sin tallo conectivo vascular, similar al carcinoma seroso de tipo ovárico.

Se administraron 4 ciclos de quimioterapia a base de carboplatino y gencitabina evitándose cisplatino por la afectación cardíaca. Durante el tratamiento presentó varias infecciones urinarias y cuadros de anemia y leucopenia que precisaron transfusiones sanguíneas y factores estimulantes de colonias (filgastrim).

Después de la QMT llevó varios meses de calidad de vida aceptable, los controles de imagen mediante ecografia y TAC a 3, 6 y 9 meses presentaron evolución satisfactoria de ambos riñones y la neovejiga. A los 10 meses inició cuadro de dolores óseos y abdominal precisando analgesia de forma continuada. En la TAC realizada al año de la intervención se evidenciaron imágenes nodulares sugestivas de recidiva mesentérica en hemiabdomen izquierdo y dilatación renoureteral bilateral. $\mathrm{El}$ paciente fallece a los 14 meses del diagnóstico tras deterioro progresivo de la función renal y el estado general por progresión intra y retroperitoneal.

\section{DISCUSIÓN}

$\mathrm{El}$ carcinoma transicional de vejiga representa más del 90\% de las neoplasias vesicales pero se han descrito una serie de variantes anatomopatológicas con implicaciones clínicas: carcinoma sarcomatoide, carcinoma de células pequeñas, carcinoma transicional con infiltrado linfoide prominente o carcinoma linfoepitelioma-like, carcinoma microquístico, carcinoma en nidos, etc. Amin y cols. ${ }^{1}$ describieron el carcinoma micropapilar de vejiga (CMP) a partir de la observación anatomoclínica en 18 pacientes. Esta variante presenta rasgos morfológicos similares al carcinoma seroso papilar de ovario.
El CMP no se trata de un tipo de lesión específico de vejiga sino que se puede localizar en otros órganos, habiéndose observado en ovario, endometrio, mama, pulmón ${ }^{2}$, vejiga y recientemente también en ureter ${ }^{3,4}$.

Tras la descripción de Amin se han comunicado varios casos clínicos aislados ${ }^{5-7}$ y pequeñas series de pacientes entre las que destacan la de Johansson $^{8}$ (20 casos), Samaratunga ${ }^{9}$ (20 casos), Lopez $^{10}$ (8 casos) y Maranchie ${ }^{11}$ (5 casos).

Amin ${ }^{1}$ describe las características histológicas de esta variante al estudiar las muestras de su grupo de pacientes y observan dos morfologías distintas según se afecte la parte superficial o la profunda. En superficie se manifiestan como procesos finos, delicados, filiformes a menudo con un núcleo central fibrovascular o bien pequeños acúmulos papilares de células tumorales; en la porción profunda o infiltrante y frecuentemente en las metástasis, las células forman pequeños nidos compactos o lagunas, que simulan infiltración vascular. Las células parecen residir en espacios huecos debido a la capacidad de penetrar y remplazar el estroma normal y lograr una extensión no endotelial. La invasión de vasos linfáticos es muy frecuente en las áreas micropapilares por lo que la tendencia a la diseminación ganglionar es habitual. Estudios inmunohistoquímicos ${ }^{1,6}$ tras tinción con CD31 y CD34 muestran no estar constituidas por células endoteliales y pueden diferenciar esos núcleos tumorales de las verdaderas invasiones vasculares, que por otra parte son muy frecuentes ${ }^{1,8}$.

Estos tumores generalmente están constituidos por componente micropapilar y componente no micropapilar en distintas proporciones ${ }^{8}$, ya sea carcinoma transicional clásico (generalmente de alto grado), carcinoma in situ , adenocarcinoma, carcinoma indiferenciado, carcinoma trofoblástico vesical ${ }^{12}$, carcinosarcoma vesical ${ }^{13}$, adenocarcinoma in situ de vejiga ${ }^{14}$, etc. $\mathrm{El}$ componente micropapilar del tumor primario, del origen que sea, suele determinar la mayor agresividad. A nivel de las metástasis puede hallarse como único componente o bien estar constituidos por más elementos.

Clínicamente presenta baja incidencia, oscilando en las distintas series entre $0,2 \% 15,1,51 \%^{10}$ y $2,2 \%^{11}$. Se presenta en edades comprendidas entre 
los 45 y los 92 años, con una media que ronda los 66 años. Respecto al sexo, predomina en varones en proporciones que oscilan entre $2,3 / 1$ en la de Johansson ${ }^{8}, 4 / 1^{9} ; 5 / 1^{1}, 5 / 0$ en los casos presentados por Maranchie ${ }^{11}$.

No presenta signos clínicos distintivos de los carcinomas clásicos de vejiga. Se han comunicado casos presentados de forma atípica como perforación vesical espontánea tras una cistoscopia en paciente con neoplasia de alto grado y bajo estadio en el que tras cistectomía se comprobó la existencia de CMP y neumatosis de pared vesical ${ }^{16}$; metástasis del conducto biliar por CMP simulando un colangiocarcinoma primario $^{17}$ y fracaso hepático por infiltración metastásica de CMP de vejiga ${ }^{18}$.

El análisis citológico de orina muestra las características de los carcinomas de alto grado; ocasionalmente en casos de carcinoma micropapilar focal pueden presentar citologías grado $2^{1}$. Ylagan $^{15}$ comunica los hallazgos citológicos en 3 de los 6 pacientes diagnosticados histológicamente de CMP destacando como signos más sugestivos:

- En estadios iniciales formaciones micropapilares que plantean diagnóstico diferencial con carcinomas de bajo grado, casos de microlitiasis o tras instrumentación, pero con intensa atipia.

- Estructuras papilares con anomalías nucleares muy llamativas: desproporción núcleo citoplasmática, grandes núcleos con membrana nuclear irregular y cromatina distribuida de forma granular hipercromática.

- Frecuentes células aisladas con atipias de alto grado.

- Grandes vacuolas tanto en células aisladas como en acúmulos celulares en nidos.

- El hallazgo de grupos celulares pequeños, uniformes y con intensa atipia citológica nos deben hacer pensar en este tipo de tumor y acelerar la confirmación y tratamiento ya que habitualmente se diagnostica en estadios avanzados ${ }^{7}$.

En nuestro caso el estudio citológico se informó como positivo para células uroteliales sugestivas de carcinoma de alto grado, sin referencia especial a CMP.

Desde el principio se ha asociado la presencia de componente micropapilar con tendencia a progresión local y a distancia - ganglios o vísceras- mayor que los carcinomas transicionales sin componente micropapilar. Su hallazgo en tumores superficiales obliga a descartar infiltración muscular en aquellos casos en los que la muestra de músculo detrusor no fuera lo suficientemente concluyente.

En ocasiones es difícil determinar el origen primario cuando se localiza en varios focos, pudiendo los estudios inmunohistoquímicos ayudar a determinar el foco inicial. En un caso clínico presentado por Ramalingan ${ }^{19}$ las biopsias de vejiga y endometrio fueron positivas para CMP pero la inmunohistoquímica indujo a considerar la mama como el origen primario y las otras localizaciones como metástasis. La paciente en cuestión tenía antecedentes de mastectomía bilateral y al analizar las muestras de vejiga, endometrio y revisar las extensiones de las mastectomías presentaban positividad para CK-7 y receptores estrogénicos y negativa para CK-20 lo que indujo a considerar las localizaciones en endometrio y vejiga como metástasis del componente micropapilar observado en mama.

La positividad para factor tiroideo de transcripción sugiere origen pulmonar; CK-7 y receptor estrogénico sugieren origen mamario mientras que la positividad para CK-7 y CK-20 sugieren origen vesical. Para Samaratunga ${ }^{9}$ la inmunotinción con CK-7 fue similar en el CMP y el carcinoma urotelial típico siendo intensa y difusa mientras que CK-20, EMA y CEA presentaba tinción variable en todos los casos.

La expresión de algunos marcadores moleculares como el p53, c-erb-2, con potencial implicación pronóstica es contradictoria. López 10 detecta positividad para estos marcadores en $7 / 8$ casos de CMP $(87,5 \%)$, siendo los resultados similares a los hallados en carcinomas de células transicionales de alto grado. Maranchie ${ }^{11}$ no demuestra alteraciones significativas del p53 en sus casos.

El caso aquí presentado expresaba positividad para CK-7, CK-20, BX2 y P53.

El tratamiento óptimo del CMP de vejiga se desconoce. A veces dado el mal estado general sólo se recurre a RTU de lesiones en estadío T1. Si están en estado general aceptable se debe recurrir a cistectomía radical incluso en estadío T1 dado que no se tiene la seguridad de una exé- 
resis completa mediante RTU teniendo en cuenta la alta incidencia de lesiones asociadas de Ca.i.s. Por otra parte la RTP y la QMT no han demostrado ser efectivas hasta la fecha. Curiosamente la supervivencia más prolongada en la serie de Johansson se produjo en un caso de cistectomía parcial asociada a radioterapia que alcanzó los 156 meses; otro paciente sometido únicamente a dos RTU permanecía vivo a los 84 meses del diagnóstico. Ahora bien, todas las evoluciones satisfactorias coincidían con tumores en estadío T1. Los pacientes tratados con QMT basada en Cisplatino no lograron resultados satisfactorios. La administración de Paclitaxel tiene que demostrar la esperanza puesta en él pero la casuística todavía es escasa dada la rareza del tumor. El paciente aquí presentado fue tratado con carboplatino y gencitabina.

El pronóstico del CMP es malo según se desprende de los datos publicados. En la serie de Amin $^{1}, 17 / 18$ se diagnosticaron en estadio avanzado B o superior, todos eran grado 3 y 7/18 habían fallecido por la enfermedad, 4 permanecían vivos con enfermedad y 7/18 no presentaban evidencia de enfermedad en el momento de la publicación tras un corto periodo de control. Johansson $^{8}$ en su serie de 20 pacientes observó la evolución durante un mínimo de 7 años y comprobó el mal pronóstico con sólo 5/20 casos vivos a los 5 años y de ellos uno murió a los 7 años por recidiva; es decir sólo 4 presentaban larga supervivencia. En el grupo de Maranchie ${ }^{11}$ en 2/5 se produjo progresión rápida de la enfermedad de forma singular, con mucosa respetada pero con invasión local de pared y a través de las vainas fibrovasculares de uréteres hacia riñón, sin afectar mucosa de uréter. El hecho de respetar mucosa de vejiga y uréter lo diferencia del carcinoma transicional de forma que las citologías y las cistoscopias pueden ser normales; asimismo pueden resultar insensibles a los tratamientos tópicos intravesicales. Por ello la presencia de componente micropapilar debe incitar a controles con biopsias en profundidad.

Santos-Briz ${ }^{6}$ presenta el caso de un paciente de 82 años con hallazgo casual de tumor vesical durante adenomectomía prostática que se trató mediante cistectomía parcial. El diagnóstico fue de CMP infiltrante asociado a carcinoma transi- cional de alto grado. El paciente se encontraba sano a los 20 meses sin tratamiento complementarios.

Samaratunga $^{9}$ estratifica los resultados anatomopatológicos de las piezas de cistectomía según el porcentaje de componente micropapilar: focal: <10\% (6 casos); moderado: 10-50\% (10 casos); extenso: $>50 \%$ (4 casos) y los correlaciona con el estadio y el pronóstico. Todos los casos con componente CMP extenso presentaban estadios T3-T4 y murieron por la enfermedad $u$ otra causa; $80 \%$ de los moderados presentaban alto estadio pT3-pT4 y 20\% pT2; de ellos 50\% habían fallecido o presentaban enfermedad; 84\% con lesión focal presentaban bajo estadio pTa-pT1.

Concluyen que el CMP muestra alto grado y el pronóstico se relaciona con la proporción y localización del componente micropapilar, siendo los casos con afectación focal y superficial los que tienen mayor probabilidad de diagnóstico precoz.

En la evolución se han descrito metástasis en peritoneo, mesenterio, ganglios retroperitoneales, cerebro, parótida, hígado, escroto, piel ${ }^{20}$, etc. El primer caso descrito de CMP de uréter ${ }^{3}$ fallece por metástasis peritoneales a los 20 meses del diagnóstico

El caso que presentamos de CMP confirma la tendencia agresiva de este tipo de tumores a pesar del tratamiento quirúrgico radical y quimioterapia sistémica, falleciendo a los 14 meses por progresión peritoneal y retroperitoneal.

En definitiva, la variante micropapilar del carcinoma de células transicionales representa una expresión celular clonal más agresiva que el carcinoma urotelial convencional. Por otro lado el aspecto microscópico y el perfil inmunohistoquímico inducen a sugerir que el carcinoma micropapilar de vejiga pudiera corresponde a una forma de adenocarcinoma de vejiga.

\section{REFERENCIAS}

1. Amin MB, Ro JY, El-Sharkawy T, Lee KM, Troncoso P, Silva GE, Ordoñez NG, Ayala GA. Micropapillary variant of transitional cell carcinoma of the urinary bladder. Histologic pattern resembling ovarian papillary serous carcinoma. Am J Surg Pathol 1994;18(12):1224-1232.

2. Amin MB, Tomboli P, Merchant SH, Ordonez NG, Ro J, Ayala AG. Micropapillary component in lung adenocarcinoma: a distinctive histologic feature with possible prognostic significance. Am J Surg Pathol 2002;26(3):358-364.

3. Oh YL, Kim KR. Micropapillary variant of transitional cell carcinoma of the ureter. Pathol Int. 2000;Jan 50(1):52-56. 
4. Vang R., Abams A. Micropapillary variant of transitional cell carcinoma arising in the ureter. Arch Pathol Lab Med 2000 sep; 124(9): 1347-1348.

5. Lopez JI, Elorriaga K, Imaz I, Bilbao FJ. Micropapillary transitional cell carcinoma of the urinary bladder. Histopathology 1999 Jun;34(6):561-562.

6. Santoz-Briz A, Antunez P, Serrano A, Ballestin C, Usera G. Carcinoma micropapilar de vejiga: presentación de un caso y revisión de la literatura. Rev Esp Patol 2001;34(2): 153-156.

7. Suarez A, Benito A, Santos-Briz A, Perez Barrios A, Alberti N, Usera G, De Agustín P. Carcinoma micropapilar infiltrante de vejiga: descripción de dos casos y hallazgos citológicos. V Congreso Virtual Hispanoamericano de Anatomía Patológica. 2002.

8. Johansson SL, Borghede G, Holmäng S. Micropapilllary bladder carcinoma: A clinicopathological study of 20 cases. J.Urol 1999;161:1798-1802.

9. Samaratunga H, Khoo K. Micropapillary variant of urothelial carcinoma of the urinary bladder: A clinicopathological and immunohistochemical study. (Abstract). Mod Pathol 2003; 16(1): 168A.

10. Lopez JI, Andrés L, Etxegarai L, Ereño C, Bilbao FJ. Micropapillary transitional cell carcinoma of the urinary bladder. A study of 8 cases. (Abstract). Mod Pathol 2003; 16 (1): 160A.

11. Maranchie JK, Bouyounes BT, Zhang PL, O'donnell MA Summerhayes IC, Dewolf WC. Clinical and pathological characteristics of micropapillary transitional cell carcinoma: a highly aggressive variant. J Urol. 2000 Mar;163(3): 748-51.

12. Regalado JJ. Mixed micropapillary and trophoblastic carcinoma of bladder: report of a first case with new immunohistochemical evidence of urothelial origin. Human Pathol. 2004 Mar;35(3):382-384.

13. Baschinsky DY, Chen JH, Vadmal MS, Lucas JG, Bahnson RR, Niemann TH. Carcinosarcoma of the urinary bladder -An aggressive tumor with diverse histogenesis. A clinicopathologic study of 4 cases and review of the literature. Arch Pathol Lab Med. 2000;124:1172-1178.
14. Chan TY, Epstein JI: In situ adenocarcinoma of the bladder. Am J Surg Pathol 2001 Jul; 25(7): 892-899.

15. Ylagan LR, Humphrey PA. Micropapillary variant of transitional cell carcinoma of the urinary bladder. A report of three cases with cytologic diagnosis in urine specimens. Acta Cytol 2001;45:599-604.

16. Kravchick S, Cytron S, Lobik L, Altshuler A, Kravchenko Y, Ben-Dor D. Clot retention and spontaneous rupture with secondary pneumatosis of the bladder wall following routine cystoscopy. Pathol Oncol Res 2001;7(4):301-302

17. Hong SP, Park SW, Lee SJ, Chung JP, Song SY, Chung JB, Kang JK, Cho NH. Bile duct wall metastasis from micropapillary variant transitional cell carcinoma of the urinary bladder mimicking primary hilar cholangiocarcinoma. Gastrointest Endosc 2002;56:756-760.

18. Baty V, Michalet V, Taniere P, Chalabreysse P, Magnin B, Ray-Coquard I. Peritoneal carcinomatosis and hepatic infiltration related to the disemination of a micropapillary cell carcinoma of the bladder. Gastroenterol Clin Biol 2003; Aug-Sep;27(8-9):828-832.

19. Ramalingam P, Middleton LP, Tomboli P, Troncoso P, Silva $\mathrm{EG}$, Ayala AG. Invasive micropapillary carcinoma of the breast metastatic to the urinary bladder and endometrium: Diagnostic pitfalls and review of the literature of tumor with micropapillary features. Ann Diagn Pathol 2003 Apr;7(2): 112-119.

20. Dominici A, Nesi G, Mondaini N, Amorosi A, Rizzo M. Skin involvement from micropapillary bladder carcinoma as the first clinical manifestation of metastatic disease. Urol Int. 2001;67(2):173-174.

Dr. L. Ripa Saldías

C/ Lezaun, 14

31620 Gorraiz (Navarra)

(Trabajo recibido el 28 de julio 2004) 